\title{
Short- and medium-term efficacy of sodium glucose cotransporter 2 (SGLT-2) inhibitors for the treatment of type 1 diabetes: systematic review and meta-analysis
}

\author{
Yuxin Huang ${ }^{1}$, Zeju Jiang ${ }^{1}$, Yiping Wei ${ }^{2}$ \\ ${ }^{1}$ Department of Clinical Medicine, The First Clinical Medical College, Nanchang University, Nanchang, China \\ ${ }^{2}$ Department of Thoracic Surgery, The Second Affiliated Hospital of Nanchang University, Nanchang, China
}

\begin{abstract}
Introduction: Sodium glucose cotransporter 2 (SGLT2) inhibitors are insulin-independent and glucose-dependent anti-hyperglycaemic drugs that have shown potential as an adjuvant therapy to insulin for the treatment of type 1 diabetes mellitus (T1DM). The purpose of this meta-analysis is to systematically collect available data from randomised trials to determine SGLT-2 inhibitor efficacy in terms of glycaemic control, body mass index, and renal protection when compared with placebo.

Material and methods: Cochrane Library, MEDLINE, and EMBASE databases were searched for randomised controlled trials and metaanalyses (without language restrictions) conducted from January 2010 to September 2019.

Results: Seventeen randomised controlled trials with 7325 participants were included. Sodium glucose cotransporter 2 therapy significantly reduced the level of glycated haemoglobin $\left(\mathrm{HbA}_{1 \mathrm{c}}\right)$ (by $0.37 \%$ ), body weight (by $2.88 \mathrm{~kg}$ ), and estimated glomerular filtration (eGFR) (by $0.67 \mathrm{~mL} / \mathrm{min} / 1.73 \mathrm{~m}^{2}$ ) when compared with placebo (all outcomes, $\mathrm{p}<0.00001$ ). Subgroup analysis by $\mathrm{HbA}_{1 \mathrm{c}}$ levels showed significant differences between six and 12 months of treatment $(\mathrm{p}<0.1)$. The magnitude of the $\mathrm{HbA}_{1 c}$ lowering effect waned with longer duration of treatment after six months (up to 12 months). Subgroup analysis by body weight showed significant differences between 1 and 3-4 months of treatment $(p<0.1)$. Weight loss plateaued after 3-4 months of treatment; subsequently, the weight remained relatively stable until 12 months. Subgroup analysis by eGFR showed significant differences between six and 12 months of treatment $(p<0.1)$. The magnitude of the eGFR lowering effect increased with longer duration of treatment after six months (up to 12 months).

Conclusions: Sodium glucose cotransporter 2 inhibitors show significant therapeutic effects when compared with placebo. Although changes in $\mathrm{HbA}_{1 c^{\prime}}$ body weight, and eGFR vary during treatment, the therapeutic effects of SGLT-2 inhibitors measured by these three outcomes can last up to 12 months. More long-term, randomised trials and extended studies are needed to determine the long-term effects of SGLT2 inhibitors as adjuvant therapy for T1DM patients. (Endokrynol Pol 2020; 71 (4): 325-333)
\end{abstract}

Key words: sodium-glucose co-transporter 2; therapeutic effects; type 1 diabetes; meta-analysis; systematic review

\section{Introduction}

Type 1 diabetes mellitus (T1DM) is an autoimmune disease characterised by chronic hyperglycaemia due to an absolute deficiency of endogenous insulin and progressive metabolic alterations that increase the risk of cardiovascular diseases [1]. Type 1 diabetes affects about 22 million adults and 400,000 children, accounting for less than $5 \%$ of the total diabetes cases in the world [2]. Insulin replacement is still the main therapy for type 1 diabetes. Compared with conventional therapy, initial intensive therapy for diabetes has been shown to result in a moderate reduction in all-cause mortality [3]. However, intensive insulin therapy to achieve strict blood sugar control is usually associated with hypoglycaemia, and insulin cannot solve other important abnormalities associated with type 1 diabetes, such as glucagon imbalance and increased gastric emptying rate (resulting in large and rapid fluctuations of postprandial blood glucose levels) [4]. In addition, insulin therapy itself is associated with weight gain and related hypertension [5]. Therefore, there is a need for adjuvant therapy that can improve key parameters continuously (such as glycated haemoglobin $\left(\mathrm{HbA}_{1 \mathrm{c}}\right)$, weight, and length of time in which the correct blood glucose range is maintained) and which can reduce the hypoglycaemic risk.

Sodium glucose cotransporter 2 (SGLT2) inhibitors are a new type of widely studied therapeutic adjuvants that can improve blood glucose control without relying on insulin. Sodium glucose cotransporter 2 inhibitors work by blocking glucose reabsorption in the renal proximal tubules, increasing urinary glucose excretion and heat and weight loss [6]. The safety and efficacy of SGLT-2 inhibitors for the treatment of type 2 diabetes have been confirmed in many studies [7-10]. On the 
other hand, many studies of combined treatment of type 1 diabetes with SGLT-2 inhibitors and insulin have shown that SGLT-2 inhibitors effectively control blood sugar levels, reduce insulin demand, and promote weight loss. However, potential side effects, such as diabetic ketoacidosis and infection, have attracted widespread scrutiny [11-14]. So far, the U.S. Food and Drug Administration (FDA) and the European Medicines Agency (EMA) have approved four SGLT2 inhibitors (canagliflozin, dapagliflozin, empagliflozin, and ertugliflozin). In addition, the double SGLT1/SGLT2 inhibitor sotagliflozin has been approved by the EMA, and in Japan another three SGLT2 inhibitors (ipraglflozin, luseoglflozin, tofogliflozin) have been approved [28].

Previous meta-analyses [11-14] have confirmed the effectiveness of SGLT-2 inhibitors as adjuvant drugs. However, these reports did not analyse the effects of these drugs at different time points in detail. In addition, the duration of most registered trials is less than or equal to 24 weeks, but the therapeutic effects of drugs may differ when the observation period is prolonged. Therefore, we conducted a meta-analysis to determine the magnitude and persistence of the therapeutic effects of SGLT-2 inhibitors as auxiliary drugs when compared with placebo, focusing mainly on three outcomes: blood glucose control, body mass index, and renal protection.

\section{Material and methods}

\section{Data sources and search}

This study followed the preferred reporting items for systematic review and meta-analysis protocols (PRISMA-P) 2015 guidelines [15]. We searched the MEDLINE, Embase, Cochrane Library (January 2010-October 30,2019), and clinical trial.gov databases, as well as online reports of major diabetes conferences, for randomised controlled trials comparing the efficacy of SGLT-2 versus placebo in adult type 1 diabetic patients. We also checked the reference lists of the original studies and reviewed articles and meta-analyses downloaded electronically, to identify other qualifying reports. There were no language restrictions. The search terms were: "Dapagliflozin OR Empagliflozin OR Sotagliflozin OR Canagliflozin OR SGLT2 OR Sodium-Glucose Cotransporter 2 Inhibitor OR SGLT2 Inhibitor OR ipragliflozin OR Sodium Glucose Transporter 2 Inhibitors OR SGLT2 Inhibitors" AND "Diabetes Mellitus, Type 1 [Mesh] OR Diabetes Mellitus, Type I OR Type 1 Diabetes OR Diabetes Mellitus, KetosisProne OR Diabetes, Autoimmune OR Diabetes Mellitus, JuvenileOnset OR Juvenile-Onset Diabetes OR Diabetes Mellitus, InsulinDependent OR IDDM OR Diabetes Mellitus, Insulin-Dependent, 1 OR Brittle Diabetes Mellitus OR Diabetes Mellitus, Sudden-Onset".

\section{Study selection}

Eligible randomised trials had to meet the following criteria:

- they compared the efficacy and safety of SGLT2 inhibitors with placebo;

- they were randomised controlled trials written in English or non-English languages

- they compared SGLT2 inhibitors with placebo as adjunctive therapy to insulin for T1DM in patients aged 18 years or above, regardless of participant gender or race.
Other therapeutic, not double-blind, non-randomised studies, or those that involved non-human species, were excluded. Studies involving children and observational studies were also excluded.

\section{Outcome measures}

The main outcome measures of this analysis were: glycated haemoglobin $\left(\mathrm{HbA}_{1 \mathrm{c}}\right)$ levels, body weight, and estimated glomerular filtration rate (EGFR) after 1, 3-4, 6, and 12 months of treatment, compared with placebo.

\section{Data extraction and risk of bias assessment}

Y.X. Huang and Z.J. Jiang performed data extraction and risk of bias assessment, respectively, and independently extracted the data in duplicate according to the Cochrane Intervention System Evaluation Manual, using a pre-designed data collection form. The extracted data included the following: the first author, year of publication, country, study population size, research and design, baseline characteristics of participants, and details of the experimental and control interventions (including dose and follow-up times). Any disputes were settled by consensus. The quality of the randomised controlled trials was assessed by means of the Cochrane bias risk tool. The bias risk map and bias summary risk for this study are shown in the Supplementary Data Figure S1 and S2. [16].

\section{Data synthesis and analysis}

The analysis was conducted using RevMan5.3 and Stata V.14.0 software, following the Cochrane Manual for Systematic Evaluation of Interventions [16], and was reported according to PRISMA Guidelines [17] (Preferred Reporting Item for Systematic Evaluation and Meta-analysis). For continuous variables, we extracted the average difference between the intervention and control groups, and the corresponding standard deviation from baseline. When the standard deviation was not reported, it was obtained from the standard error, as described in the Cochrane manual. When only the difference between the average values of the two groups was reported, the standard deviation was also calculated according to the Cochrane Manual [16]. Heterogeneity was evaluated based on $\mathrm{I}^{2}$ statistics. We used regression analysis to explore the reasons for results showing large heterogeneity [18]. Publication bias was visually assessed by drawing funnel plots and by performing Egger weighted regression tests [19]. We conservatively used the prior random effects model, assuming there were significant differences in therapeutic effects between studies.

\section{Results}

\section{Literature retrieval and study selection}

A database search led to the identification of 2711 eligible research records, 298 of which were excluded due to duplication. After screening the titles and abstracts of the remaining 2413 studies, a total of 2386 studies were excluded based on the inclusion and exclusion criteria: 24 were duplicates, 1756 involved type 2 diabetes, 134 were animal experiments, 441 had no original data, and 31 were unrelated. Finally, a total of 27 full articles were considered for further analysis. Because 10 of these were duplicates, a total of 17 studies evaluating the efficacy of SGLT2 combined with insulin therapy in patients with type 1 diabetes were included. The articles written by Dandona 2017 and Dandona 2018 were about the same study but differed in the follow-up duration 


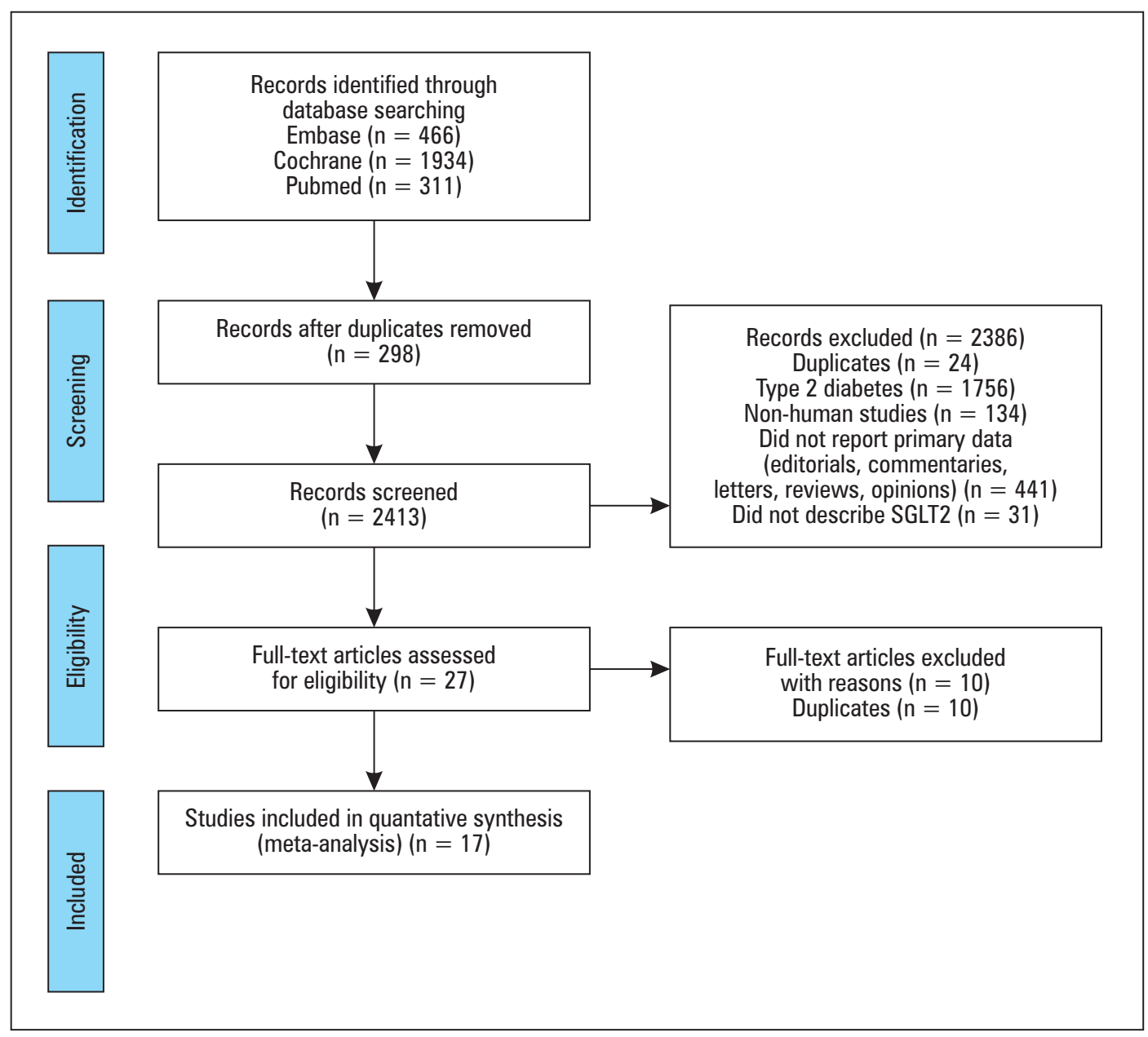

Figure 1. Flowchart of the literature screening

(24 and 52 weeks, respectively). The entire process is summarised in Figure 1.

\section{Research characteristics}

All the studies included in this meta-analysis were double-blind, randomised controlled trials and were published as full-length articles from 2014 to 2018. The follow-up time in individual studies ranged from 1 week to 52 weeks. Baseline characteristics were well balanced in the individual studies. A placebo was administered in the control arm in all trials (Tab. 1).

\section{Search results and quality assessment}

\section{Glycated haemoglobin $\left(\mathrm{HbA}_{1 \mathrm{c}}\right)$}

The meta-analysis revealed that SGLT2 therapy significantly reduced $\mathrm{HbA}_{1 \mathrm{c}}$ levels by $0.37 \%$ (weighted mean difference [WMD]: $-0.37 \%$; 95\% confidence interval [CI]: $\left.-0.41,-0.33 ; \mathrm{I}^{2}=99 \% ; \mathrm{p}<0.00001\right)$. The subgroup analysis indicated that all subgroups showed statistical significance, including the 1-month subgroup (WMD: $-0.43 \%$; 95\% CI: $-0.59,-0.27 ; \mathrm{I}^{2}=0 \%$; $\left.<<0.00001\right)$, the 3-4-months subgroup (WMD: $-0.38 \%$; 95\% CI: $-0.51,-0.25 ; \mathrm{I}^{2}=91 \%$; $\left.<<0.00001\right)$, the 6 -months subgroup (WMD: $-0.40 \%$; 95\% CI: $-0.44,-0.36$; $\mathrm{I}^{2}=99 \%$; $\mathrm{p}<0.00001$ ), and the 12-months subgroup (WMD: $-0.30 \%$; 95\% CI: $-0.35,-0.26$; $\mathrm{I}^{2}=98 \%$; $\left.<<0.00001\right)$. Moreover, the 1-month subgroup showed no significant difference with the 3-4-months subgroup. Similarly, the 3-4-months subgroup showed no significant difference with the 6-months subgroup. However, there was a significant difference between the 6-months subgroup and the 12-months subgroup ( $\mathrm{p}<0.1$ ). The magnitude of the $\mathrm{HbA}_{1 \mathrm{c}}$ lowering effect waned with longer duration of treatment after six months (up to 12 months) (Fig. 2).

\section{Body weight}

The meta-analysis revealed that SGLT2 therapy significantly reduced body weight by $2.88 \mathrm{~kg}$ (WMD: -2.88 ; 95\% CI: $-3.11,-2.65 ; \mathrm{I}^{2}=99 \%$; $\left.\mathrm{p}<0.00001\right)$. The subgroup analysis indicated that all subgroups showed statistically significant differences with placebo, including the 1-month subgroup (WMD: -1.77 ; 95\% CI: $\left.-1.93,-1.61 ; I^{2}=0 \% ; p<0.00001\right)$, the 3-4-months 


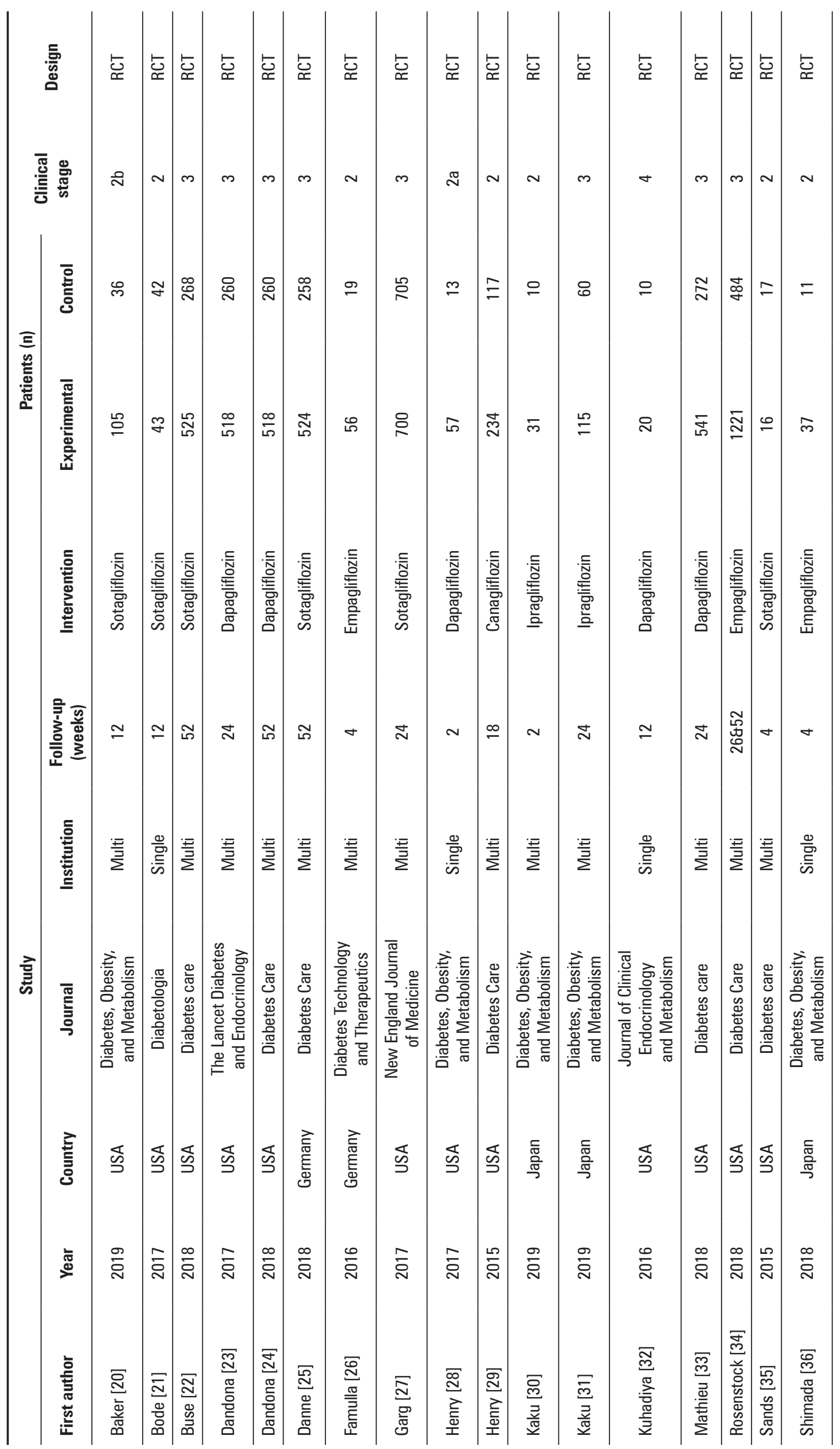




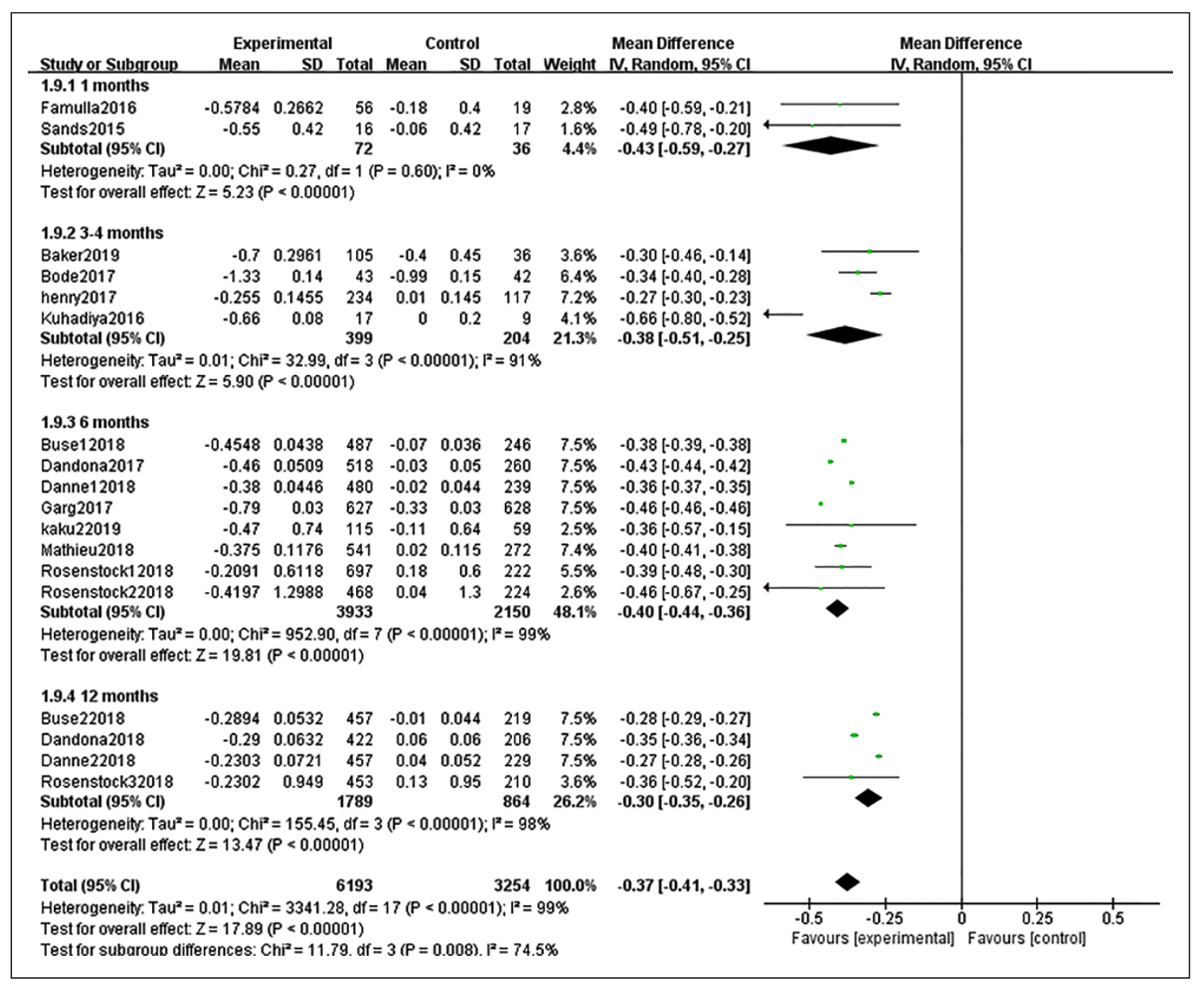

Figure 2. Forest plot of mean difference in glycated haemoglobin ( $\left.\mathrm{Hb} \mathrm{A}_{1}\right)$. Horizontal lines represent the $95 \%$ confidence interval (CI). Solid squares indicate the mean difference and are proportional to the weights used in the meta-analysis. Solid vertical line indicates no effect. Diamond indicates the weighted mean difference (WMD); the lateral tips of the diamond indicate the associated 95\% CI

subgroup (WMD: $-2.83 ; 95 \% \mathrm{CI}:-3.90,-1.76 ; \mathrm{I}^{2}=86 \%$; $\mathrm{p}<0.00001$ ), the 6-months subgroup (WMD: -2.87 ; 95\% CI: $\left.-3.19,-2.55 ; \mathrm{I}^{2}=100 \% ; \mathrm{p}<0.00001\right)$, and the 12-months subgroup (WMD: -3.21 ; 95\% CI: -3.86 , $\left.-2.55 ; \mathrm{I}^{2}=100 \% ; \mathrm{p}<0.00001\right)$. Moreover, the 1 -month subgroup showed a significant difference with the 3-4-months subgroup ( $\mathrm{p}<0.1$ ), but the 3-4-months subgroup showed no significant difference with the 6-months subgroup. Similarly, there was no significant difference between the 6-months subgroup and the 12-months subgroup. Weight loss plateaued after 3-4 months of treatment, but thereafter the weight remained relatively stable and was maintained until 12 months (Fig. 3).

\section{Estimated glomerular filtration rate (eGFR)}

The meta-analysis revealed that SGLT2 therapy significantly reduced the eGFR by $0.67 \mathrm{~mL} / \mathrm{min} / 1.73 \mathrm{~m}^{2}$ (WMD: $-0.67 ; 95 \%$ CI: $-0.71,-0.63 ; \mathrm{I}^{2}=100 \%$; $\left.\mathrm{p}<0.00001\right)$. The subgroup analysis indicated that both the 6-months subgroup (WMD: -0.51 ; 95\% CI: $-0.55,-0.46$; $\mathrm{I}^{2}=100 \%$; $\mathrm{p}<0.00001)$ and the 12-months subgroup showed statistically significant differences with placebo (WMD: -1.32 ; 95\% CI: $-1.42,-1.23 ; \mathrm{I}^{2}=72 \%$; $\left.\mathrm{p}<0.00001\right)$. Moreover, there was a significant difference between the 6-months subgroup and the 12-months subgroup ( $p<0.1)$. Hence, the eGFR lowering effect increased with longer duration of treatment after six months (up to 12 months) (Fig. 4).

\section{Evaluated parameters in different time-points}

Figure 5 shows the reduction of $\mathrm{HbA}_{1 \mathrm{c}}$ (black line) compared with the control group in different follow-up time (1 month, 3-4 months, 6 months, 12 months); it also shows the reduction of body weight (red line) compared with the control group in different follow-up time (1 month, 3-4 months, 6 months, 12 months), and that the reduction of eGFR (blue line) compared with the control group in different follow-up time ( 6 months, 12 months). The data are represented by the table included in the figure. The analysis was performed with log-transformed values 


\begin{tabular}{|c|c|c|c|c|c|c|c|c|c|c|c|}
\hline \multirow[b]{2}{*}{ Stucty or Subgroup } & \multicolumn{3}{|c|}{ Experimental } & \multicolumn{2}{|c|}{ Control } & \multirow[b]{2}{*}{ Total } & \multirow{2}{*}{\multicolumn{2}{|c|}{$\begin{array}{c}\text { Mean Difference } \\
\text { Weight } \text { N. Random, } 95 \% \mathrm{Cl}\end{array}$}} & \multirow{2}{*}{\multicolumn{3}{|c|}{$\begin{array}{l}\text { Mean Difference } \\
\text { IV. Random, } 95 \% \mathrm{Cl}\end{array}$}} \\
\hline & Mean & SD & Total & Mean & SD & & & & & & \\
\hline \multicolumn{12}{|l|}{ 1.11.11 months } \\
\hline Famulla2016 & -1.5643 & 0.3202 & 56 & 0.2 & 0.3 & 19 & $8.1 \%$ & $-1.76[-1.92,-1.61]$ & - & & \\
\hline Sands2015 & -1.7 & 2.09 & 16 & 0.5 & 2.09 & 17 & $2.0 \%$ & $-2.20[-3.63,-0.77]$ & - & & \\
\hline Subtotal $(95 \% \mathrm{Cl})$ & & & 72 & & & 36 & $10.1 \%$ & $-1.77[-1.93,-1.61]$ & & & \\
\hline \multicolumn{12}{|c|}{$\begin{array}{l}\text { Heterogeneity. } \text { Tau }^{2}=0.00 ; \mathrm{Chi}^{2}=0.35, \mathrm{df}=1(P=0.55) ; \mathrm{I}^{2}=0 \% \\
\text { Test for overall effect: } Z=21.97(P<0.00001)\end{array}$} \\
\hline \multicolumn{12}{|l|}{ 1.11.2 3.4 months } \\
\hline Baker2019 & -0.9667 & 1.3161 & 105 & 1.1 & 2.59 & 36 & $3.8 \%$ & $-2.07[-2.95,-1.18]$ & - & & \\
\hline henry 2017 & -1.9 & 0.54 & 17 & 0.7 & 1.5 & 9 & $3.2 \%$ & $-2.60[-3.61,-1.59]$ & $\cdot$ & & \\
\hline Kuhadiya2016 & -3.4 & 1.0802 & 234 & 0.2 & 0.725 & 117 & $7.9 \%$ & $-3.60[-3.79,-3.41]$ & $\cdot 1$ & & \\
\hline Subtotal $(95 \% \mathrm{Cl})$ & & & 356 & & & 162 & $14.9 \%$ & $-2.83[-3.90,-1.76]$ & 1 & & \\
\hline \multicolumn{12}{|c|}{$\begin{array}{l}\text { Heterogeneity. Tau }{ }^{2}=0.75 ; \mathrm{Chi}^{2}=14.22, \mathrm{df}=2(P=0.0008) ; \mathrm{I}^{2}=86 \% \\
\text { Test for overall effect: } Z=5.18(P<0.00001)\end{array}$} \\
\hline \multicolumn{12}{|l|}{1.11 .36 months } \\
\hline Buse12018 & -2.1166 & 0.5817 & 487 & 0.78 & 0.187 & 244 & $8.3 \%$ & $-2.90[-2.95,-2.84]$ & $\cdot$ & & \\
\hline Dandona2017 & -3.22 & 0.455 & 518 & 0.12 & 0.26 & 260 & $8.4 \%$ & $-3.34[-3.39,-3.29]$ & $\cdot$ & & \\
\hline Danne12018 & -2.1756 & 0.3563 & 481 & 0.11 & 0.201 & 240 & $8.4 \%$ & $-2.29[-2.33,-2.24]$ & $\cdot$ & & \\
\hline Garg2017 & -2.21 & 0.12 & 630 & 0.77 & 0.12 & 633 & $8.4 \%$ & $-2.98[-2.99,-2.97]$ & $\cdot$ & & \\
\hline kaku22019 & -2.92 & 2.34 & 115 & -0.04 & 2.02 & 59 & $4.9 \%$ & $-2.88[-3.55,-2.21]$ & $\cdot$ & & \\
\hline Rosenstock1 2018 & -2.5383 & 0.8724 & 720 & 0.2 & 5 & 238 & $5.1 \%$ & $-2.74[-3.38,-2.10]$ & $\cdot$ & & \\
\hline Rosenstock22018 & -3.0981 & 0.6261 & 483 & -0.1 & 7.55 & 238 & $3.4 \%$ & $-3.00[-3.96,-2.04]$ & $\cdot$ & & \\
\hline Subtotal $(95 \% \mathrm{Cl})$ & & & 3434 & & & 1912 & $47.0 \%$ & $-2.87[-3.19,-2.55]$ & 1) & & \\
\hline \multicolumn{12}{|c|}{$\begin{array}{l}\text { Heterogeneity. Tau² }=0.14 ; \mathrm{Chi}^{2}=1270.73, \mathrm{df}=6(P<0.00001) ; \mathrm{P}^{2}=100 \% \\
\text { Test for overall effect: } Z=17.74(P<0.00001)\end{array}$} \\
\hline \multicolumn{12}{|l|}{ 1.11.4 12 months } \\
\hline Buse22018 & -2.5184 & 0.6406 & 457 & 1.2 & 0.249 & 219 & $8.3 \%$ & $-3.72[-3.79,-3.65]$ & $\cdot$ & & \\
\hline Dandona2018 & -2.98 & 0.723 & 422 & 0.25 & 0.27 & 206 & $8.3 \%$ & $-3.23[-3.31,-3.15]$ & - & & \\
\hline Danne22018 & -2.2591 & 0.4542 & 461 & 0.3 & 0.257 & 229 & $8.4 \%$ & $-2.56[-2.61,-2.51]$ & $=$ & & \\
\hline Rosenstock32018 & -3.1988 & 0.7517 & 483 & 0.2 & 7.86 & 210 & $3.0 \%$ & $-3.40[-4.46,-2.33]$ & $=$ & & \\
\hline Subtotal $(95 \% \mathrm{Cl})$ & & & 1823 & & & 864 & $28.0 \%$ & $-3.21[-3.86,-2.55]$ & 1 & & \\
\hline \multicolumn{12}{|c|}{$\begin{array}{l}\text { Heterogeneity. Tau }{ }^{2}=0.40 ; \mathrm{Chi}^{2}=726.42, \mathrm{df}=3(\mathrm{P}<0.00001) ; \mathrm{I}^{2}=100 \% \\
\text { Test for overall effect: } Z=9.54(P<0.00001)\end{array}$} \\
\hline Total $(95 \% \mathrm{Cl})$ & & & 5685 & & & 2974 & $100.0 \%$ & $-2.88[-3.11,-2.65]$ & 1 & & \\
\hline \multicolumn{9}{|c|}{$\begin{array}{l}\text { Heterogeneity. } \mathrm{Tau}^{2}=0.17: \mathrm{Chi}^{2}=2295.80, \mathrm{df}=15 \\
\text { Test for overall effect: } Z=24.33(P<0.00001) \\
\text { Test for subaroun differences: } \mathrm{Chi}^{2}=51.66 . \mathrm{df}=3\end{array}$} & $\begin{array}{lc}1 & 1 \\
-100 & -50 \\
& \text { Favours [experimental] }\end{array}$ & $\begin{array}{l}0 \\
\text { Favours [control] }\end{array}$ & $\overrightarrow{100}$ \\
\hline
\end{tabular}

Figure 3. Forest plot of mean difference in body weight. Horizontal lines represent the 95\% confidence interval (CI). Solid squares indicate the mean difference and are proportional to the weights used in the meta-analysis. Solid vertical line indicates no effect. Diamond indicates the weighted mean difference (WMD); the lateral tips of the diamond indicate the associated 95\% CI

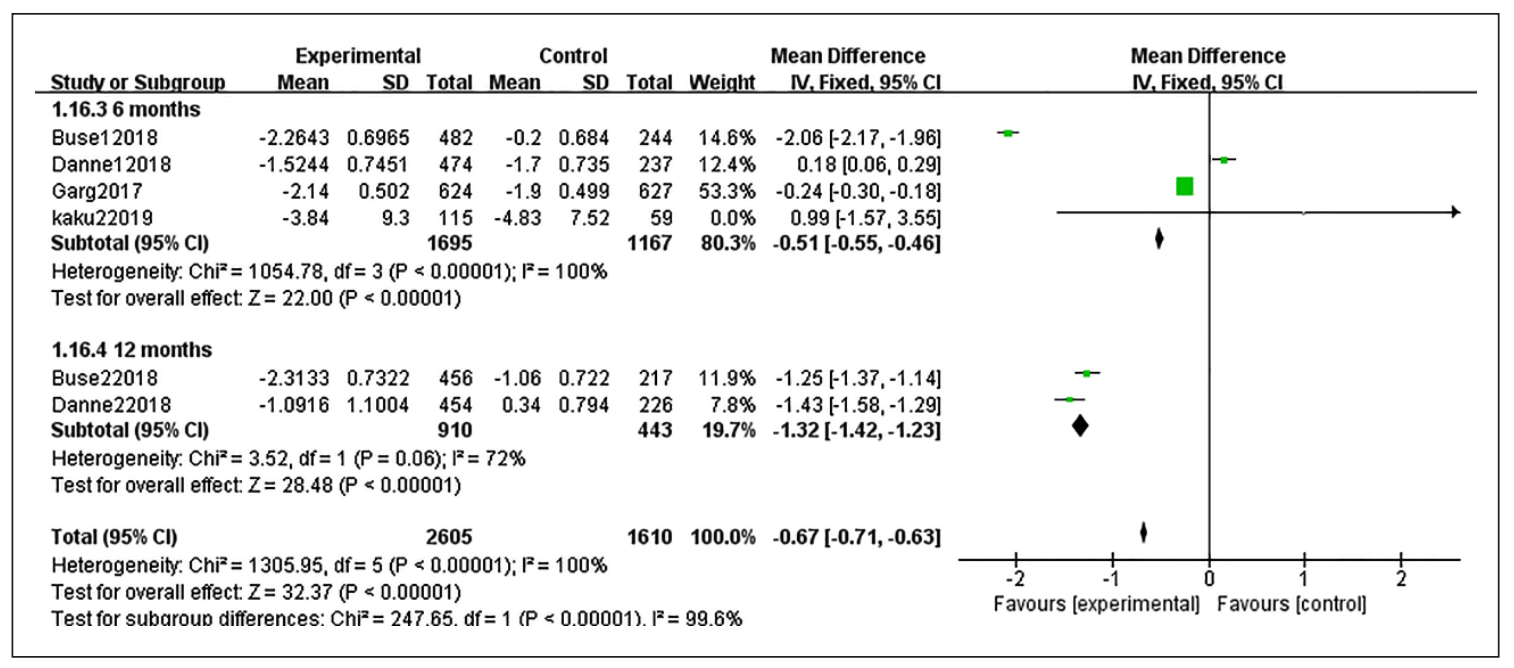

Figure 4. Forest plot of mean difference in estimated glomerular filtration rate (eGFR). Horizontal lines represent the $95 \%$ confidence interval (CI). Solid squares indicate the mean difference and are proportional to the weights used in the meta-analysis. Solid vertical line indicates no effect. Diamond indicates the weighted mean difference (WMD); the lateral tips of the diamond indicate the associated $95 \%$ CI

by analysis of covariance and corrected for the baseline values. In both panels, means and $95 \%$ confidence intervals are shown.

\section{Risk of bias in the study and meta-regression}

We used the Cochrane Risk Bias Assessment Tool included in Revman 5.3 to assess bias in the 17 randomised 


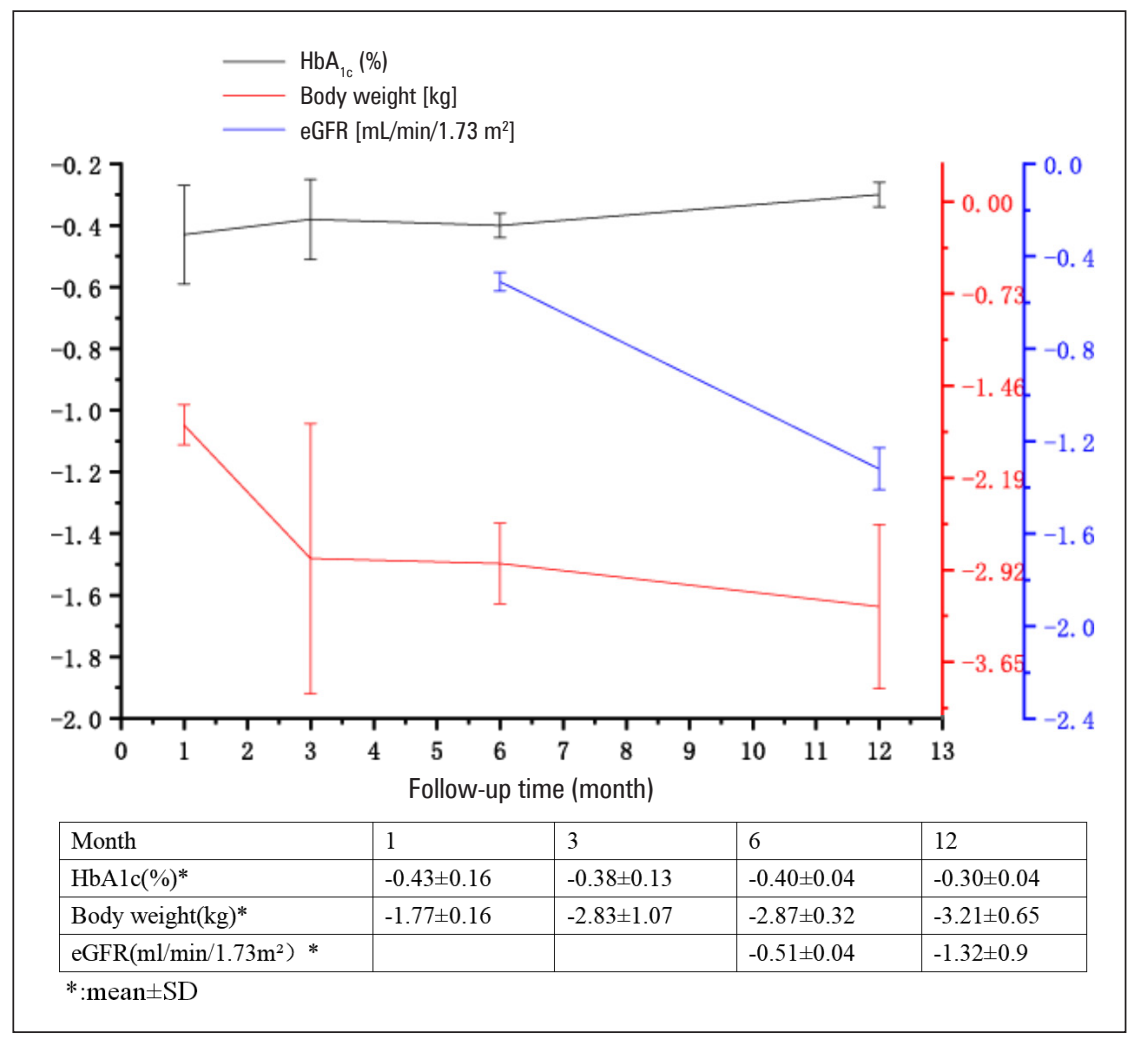

Figure 5. Evaluated parameters in different time-points. $H b A_{1 c}-$ glycated haemoglobin; eGFR — estimated glomerular filtration rate

trials and constructed a bias risk map and a bias risk summary map. We identified three studies with one high risk of bias, six studies with one or two unknown risks of bias, and eight high-quality studies. We conducted meta-regression on the outcome indicators showing large heterogeneity to explore the causes of the heterogeneity, and finally only the $\mathrm{HbA}_{1 \mathrm{c}}$ results showed statistical significance. Our meta-regression analysis detected a linear correlation between $\mathrm{HbA}_{1 \mathrm{c}}$ levels and follow-up time (regression coefficient 1.003; $\mathrm{p}=0.013$ ) (Supplementary File — Figure S3).

\section{Discussion}

Short and medium-term efficacy of sodium glucose cotransporter 2 inhibitors are a new class of antidiabetic drugs, which work independently of insulin. They have been shown to improve blood sugar control, body weight, and blood pressure in T2DM [9]. Previous studies $[12,13]$ also confirmed that SGLT2 inhibitors are effective when used to treat T1DM. Because adjuvant drug therapy for type 1 diabetes is usually long-term, it is crucial to determine the magnitude and persistence of the therapeutic effects of these agents. In this review, we summarise the data of 17 clinical trials conducted in patients with type 1 diabetes. When the results of all the placebo-controlled trials were combined, we found that although changes in $\mathrm{HbA}_{1 \mathrm{c}^{\prime}}$, body weight, and eGFR differed during the treatment period, the therapeutic effects of the SGLT-2 inhibitors, based on these three outcomes, could last up to 12 months. At present, there are no large-scale experiments longer than 12 months to determine the duration of the therapeutic effects of SGLT2 inhibitors in patients with type 1 diabetes.

Glycosylated haemoglobin $\left(A_{1 c}\right)$ is an effective surrogate marker for evaluating the success of long-term diabetes treatment. According to the U.S. Food and Drug Administration (FDA), the primary endpoint to determine the efficacy of drugs that lower blood glucose levels should be $A_{1 c}$ reduction [37]. HbA1c has been proven to be highly valuable for patient management, it is an important measure of population health, and is still an effective indicator of glycation as a complicating risk factor [38]. Controlling glycosylated haemoglobin levels reduces the side effects of insulin therapy in T1DM patients. Based on our meta-analysis, SGLT2 inhibitors show a relatively stable $\mathrm{HbA}_{1 \mathrm{c}}$ lowering effect during the first six months of treatment, but then it wanes after six months of treatment (up to 12 months).

Compared with short-term treatment, long-term treatment with SGLT2 inhibitors can lead to continuous weight loss. These findings are important. Although significant progress has been made in insulin preparation, delivery systems, and blood glucose monitoring, only one third of patients achieve the desired blood glucose target, and many patients become overweight 
or obese [39-41]. Previous evidence shows that moderate weight loss is associated with a beneficial reduction of cardiovascular risk factors [42, 43]. Losing weight may also help patients get psychological support from treatment plans. Based on our meta-analysis, weight loss plateaued after 3-4 months of treatment with SGLT2 inhibitors; thereafter, the weight was relatively stable up to 12 months.

In the early stages of diabetes, the glomerular filtration rate can increase, and this is one of the mechanisms of diabetic nephropathy. The early effects of SGLT2 inhibitors on glomerular ultrafiltration and urinary protein excretion are an indication of their potential renal protective effects [11]. Our study showed that the eGFR lowering effect increased after six months of treatment (up to 12 months). Hence, SGLT2 inhibitors as adjuvant drugs in T1DM also have long-term renal protective effects.

EI Masri et al. [13] carried out two subgroup analyses of dose and treatment duration, but only included four randomised controlled trials and only analysed the effects of dose and treatment duration on $\mathrm{HbA}_{1 \mathrm{c}}$ and body weight, two outcome indicators. $\mathrm{Xu}$ et al. [12] performed a meta-analysis, together with three gradient dose sub-analyses, of SGLT2 inhibitors in T1DM, and pointed out that more sub-analyses were needed, such as long- and short-term treatment analyses. In addition to previous studies [12], four new studies $[24,30,31,34]$ have been added to the current meta-analysis, including the experimental results of the new drug ipragliflozin $[30,31]$. Hence, this is the most comprehensive and reliable meta-analysis so far, including 17 trials. We performed subgroup analysis based on treatment duration at four time points and analysed changes in $\mathrm{HbA}_{1 \mathrm{c}^{\prime}}$, body weight, and eGFR over time. The extensive data collected in this study allows us to compare SGLT2 inhibitors and placebo at different time points, revealing the effects of SGLT-2 inhibitors over time. We also explored heterogeneity and identified the heterogeneous sources of glycosylated haemoglobin. Unfortunately, we were unable to successfully explore the heterogeneous source of other parameters. We studied the overall effects of SGLT2 inhibitors as a class of drugs, and in the process, we may have neglected to consider the influence of different drug types. The therapeutic effects may also be influenced by the characteristics of the participants in clinical trials and may differ for different molecules. In addition, most of this research is industry-funded, which may lead to further bias. We may also have missed unpublished reports. Many other reasons can explain the observed heterogeneity, and these viewpoints deserve further attention in specially designed meta-analyses.

\section{Conclusion}

This review provides information about the therapeutic effects of SGLT2 inhibitors in T1DM. More long-term randomised trials are needed to determine the long-term effects of SGLT2 inhibitors as adjuvants in the treatment of T1DM patients.

\section{Acknowledgements}

Not applicable.

\section{Funding}

This work was supported by grants from the National Natural Science Foundation of China (81560345 and 81860379), Pre-eminence Youth Fund of Jiangxi Province (20162BCB23058), China Postdoctoral Science Foundation Grant (2017M610401), Science and Technology Planning Project at the Department of Science, and Technology of Jiangxi Province, China (20151BBG70165 and 20171BAB205075). Conflicts of Interest: The authors declare no conflicts of interest.

\section{Availability of data and materials}

The analysed data sets generated during the study are available in the published article.

\section{Authors' contributions}

Y.H. and Z.J. contributed equally; Y.H. conceptualised and designed the study; Z.J. and Y.H. conducted the database search, study selection, and data extraction process; Y.W. conducted quality assessment of screened studies; Z.J. and Y.H. wrote the manuscript; Y.W. approved the final version of this manuscript and takes responsibility for its contents.

\section{Ethical approval and consent to participate \\ Not applicable.}

\section{Patient consent for publication}

Not applicable.

\section{Competing interests}

The authors declare that they have no competing interests.

\section{References}

1. Chiang J, Kirkman M, Laffel L, et al. Type 1 Diabetes Through the Life Span: A Position Statement of the American Diabetes Association. Diab Care. 2014; 37(7): 2034-2054, doi: 10.2337/dc14-1140, indexed in Pubmed: 24935775.

2. Health Canada. 2014. Summary of Health Canada'sAssessment of a Health Claim about GroundWhole Flaxseed and Blood Cholesterol Lowering. http://www.hc-sc.gc.ca/fn-an/alt_formats/pdf/label-etiquet/claims-reclam/assess-evalu/flaxseedgraines-de-lin-eng. (May 11 2014).

3. Orchard T, Nathan D, Zinman B, et al. Association Between 7 Years of Intensive Treatment of Type 1 Diabetes and Long-term Mortality. 
JAMA. 2015; 313(1): 45-53, doi: 10.1001/jama.2014.16107, indexed in Pubmed: 25562265.

4. Frandsen C, Dejgaard T, Madsbad S. Non-insulin drugs to treat hyperglycaemia in type 1 diabetes mellitus. Lancet Diabetes Endocrinol. 2016; 4(9): 766-780, doi: 10.1016/s2213-8587(16)00039-5, indexed in Pubmed: 26969516.

5. Purnell J, Hokanson J, Marcovina S, et al. Effect of Excessive Weight Gain With Intensive Therapy of Type 1 Diabetes on Lipid Levels and Blood Pressure. JAMA. 1998; 280(2): 140-146, doi: 10.1001/jama.280.2.140, indexed in Pubmed: 9669786.

6. Vasilakou D, Karagiannis T, Athanasiadou E, et al. Sodium-Glucose Cotransporter 2 Inhibitors for Type 2 Diabetes. Ann Int Med. 2013; 159(4): 262-274, do i: 10.7326/0003-4819-159-4-201308200-00007, indexed in Pubmed: 24026259

7. Yang XP, Lai D, Zhong XY, et al. Efficacy and safety of canagliflozin in subjects with type 2 diabetes: systematic review and meta-analysis. Eur J Clin Pharmacol. 2014; 70(10): 1149-1158, doi: 10.1007/s00228-014-1730-x indexed in Pubmed: 25124541.

8. Baker W, Smyth L, Riche D, et al. Effects of sodium-glucose co-transporter 2 inhibitors on blood pressure: A systematic review and meta-analysis. J Am Soc Hypertens. 2014; 8(4): 262-275.e9, doi: 10.1016/j. jash.2014.01.007, indexed in Pubmed: 24602971.

9. Monami M, Nardini C, Mannucci E. Efficacy and safety of sodium glucose co-transport- 2 inhibitors in type 2 diabetes: a meta-analysis of randomized clinical trials. Diabetes Obes Metab. 2013; 16(5): 457-466, doi: 10.1111/dom.12244, indexed in Pubmed: 24320621.

10. Liakos A, Karagiannis T, Athanasiadou E, et al. Efficacy and safety of empagliflozin for type 2 diabetes: a systematic review and meta-analysis. Diabetes Obes Metab. 2014; 16(10): 984-993, doi: 10.1111/dom.12307, indexed in Pubmed: 24766495.

11. Chen J, Fan F, Wang JY, et al. The efficacy and safety of SGLT2 inhibitors for adjunctive treatment of type 1 diabetes: a systematic review and meta-analysis. Scientific Reports. 2017; 7(1): 44128, doi: 10.1038/srep44128, indexed in Pubmed: 28276512.

12. Li K, Xu G. Safety and efficacy of sodium glucose co-transporter 2 inhibitors combined with insulin in adults with type 1 diabetes: A meta-analysis of randomized controlled trials. J Diabetes. 2019; 11(8): 645-655, doi: 10.1111/1753-0407.12890, indexed in Pubmed: 30565398.

13. Masri DEl, Ghosh S, Jaber L. Safety and efficacy of sodium-glucose cotransporter 2 (SGLT2) inhibitors in type 1 diabetes: A systematic review and meta-analysis. Diabetes Res Clin Pract. 2018; 137: 83-92, doi: 10.1016/j.diabres.2018.01.004, indexed in Pubmed: 29317332.

14. Yamada T, Shojima N, Noma H, et al. Sodium-glucose co-transporter-2 inhibitors as add-on therapy to insulin for type 1 diabetes mellitus: Systematic review and meta-analysis of randomized controlled trials. Diabetes Obes Metab. 2018; 20(7): 1755-1761, doi: 10.1111/dom.13260, indexed in Pubmed: 29451721.

15. Shamseer L, Moher D, Clarke M, et al. Preferred reporting items for systematic review and meta-analysis protocols (PRISMA-P) 2015: elaboration and explanation. BMJ. 2015; 350: g7647, doi: 10.1136/bmj.g7647, indexed in Pubmed: 25555855.

16. Higgins JPT, Green S. Cochrane handbook for systematic reviews of interventions, version 5.2.0., updated March 2011. Cochrane Collaboration, John Wiley \& Sons Ltd 2011: July.

17. Liberati A, Altman DG, Tetzlaff J, et al. The PRISMA statement for reporting systematic reviews and meta-analyses of studies that evaluate healthcare interventions: explanation and elaboration. BMJ. 2009; 339: b2700, doi: 10.1136/bmj.b2700, indexed in Pubmed: 19622552.

18. Higgins J. Measuring inconsistency in meta-analyses. BMJ. 2003, 327(7414): 557-560, doi: $10.1136 / \mathrm{bmj} .327 .7414 .557$, indexed in Pubmed: 12958120.

19. Egger M, Smith GD, Schneider M, et al. Bias in meta-analysis detected by a simple, graphical test. BMJ. 1997; 315(7109): 629-634, doi: 10.1136/bmj.315.7109.629, indexed in Pubmed: 9310563

20. Baker C, Wason S, Banks P, et al. Dose-dependent glycometabolic effects of sotagliflozin on type 1 diabetes over 12 weeks: The inTandem 4 trial. Diabetes Obes Metab. 2019; 21(11): 2440-2449, doi: 10.1111/dom.13825, indexed in Pubmed: 31264767.

21. Bode B, Banks P, Sawhney S, et al. Efficacy and safety of sotagliflozin, a dual SGLT1 and SGLT2 inhibitor, as adjunct to insulin in young adults with poorly controlled type 1 diabetes (JDRF Study). Diabetologia. 2017; 60: S87-S88.

22. Buse J, Garg S, Rosenstock J, et al. Sotagliflozin in Combination With Optimized Insulin Therapy in Adults With Type 1 Diabetes: The North American inTandem1 Study. Diab Care. 2018; 41(9): 1970-1980, doi: 10.2337/dc18-0343, indexed in Pubmed: 29937430.

23. Dandona P, Mathieu C, Phillip M, et al. Efficacy and safety of dapagliflozin in patients with inadequately controlled type 1 diabetes (DEPICT-1): 24 week results from a multicentre, double-blind, phase 3, randomised controlled trial. Lancet Diab Endocrinol. 2017; 5(11): 864-876, doi: 10.1016/s2213-8587(17)30308-x, indexed in Pubmed: 28919061.

24. Dandona P, Mathieu C, Phillip M, et al. Efficacy and Safety of Dapagliflozin in Patients With Inadequately Controlled Type 1 Diabetes:
The DEPICT-1 52-Week Study. Diabetes Care. 2018; 41(12): 2552-2559, doi: 10.2337/dc18-1087, indexed in Pubmed: 30352894

25. Danne T, Cariou B, Banks P, et al. HbA1cand Hypoglycemia Reduction at 24 and 52 Weeks With Sotagliflozin in Combination With Insulin in Adults With Type 1 Diabetes: The European inTandem2 Study. Diab Care. 2018; 41(9): 1981-1990, doi: 10.2337/dc18-0342, indexed in Pubmed: 29937431.

26. Famulla S, Pieber T, Eilbracht J, et al. Glucose Exposure and Variability with Empagliflozin as Adjunct to Insulin in Patients with Type 1 Diabetes: Continuous Glucose Monitoring Data from a 4-Week, Randomized, Placebo-Controlled Trial (EASE-1). Diab Technol Ther. 2017; 19(1): 49-60, doi: 10.1089/dia.2016.0261, indexed in Pubmed: 27929674.

27. Garg S, Henry R, Banks P, et al. Effects of Sotagliflozin Added to Insulin in Patients with Type 1 Diabetes. N Engl J Med. 2017; 377(24): 2337-2348, doi: 10.1056/nejmoa1708337, indexed in Pubmed: 28899222.

28. Henry R, Dandona P, Pettus J, et al. Dapagliflozin in patients with type 1 diabetes: Apost hoc analysis of the effect of insulin dose adjustments on 24-hour continuously monitored mean glucose and fasting -hydroxybutyrate levels in a phase IIa pilot study. Diabetes Obes Metab. 2017; 19(6): 814-821, doi: 10.1111/dom.12882, indexed in Pubmed: 28098426.

29. Henry R, Thakkar P, Tong C, et al. Efficacy and Safety of Canagliflozin, a Sodium-Glucose Cotransporter 2 Inhibitor, as Add-on to Insulin in Patients With Type 1 Diabetes. Diabetes Care. 2015; 38(12): 2258-2265, doi: 10.2337/dc15-1730, indexed in Pubmed: 26486192.

30. Kaku K, Isaka H, Toyoshima J, et al. Clinical pharmacology study of ipragliflozin in Japanese patients with type 1 diabetes mellitus: A phase 2, randomized, placebo-controlled trial. Diabetes Obes Metab. 2019; 21(6): 1445-1454, doi: 10.1111/dom.13679, indexed in Pubmed: 30821047

31. Kaku K, Isaka $\mathrm{H}$, Sakatani $\mathrm{T}$, et al. Efficacy and safety of ipragliflozin add-on therapy to insulin in Japanese patients with type 1 diabetes mellitus: A randomized, double-blind, phase 3 trial. Diabetes Obes Metab. 2019; 21(10): 2284-2293, doi: 10.1111/dom.13807, indexed in Pubmed: 31173455.

32. Kuhadiya N, Ghanim H, Mehta A, et al. Dapagliflozin as Additional Treatment to Liraglutide and Insulin in Patients With Type 1 Diabetes. J Clin Endocrinol Metab. 2016; 101(9): 3506-3515, doi: 10.1210/jc.2016-1451, indexed in Pubmed: 27490915

33. Mathieu C, Dandona P, Gillard P, et al. Efficacy and Safety of Dapagliflozin in Patients With Inadequately Controlled Type 1 Diabetes (the DEPICT-2 Study): 24-Week Results From a Randomized Controlled Trial. Diabetes Care. 2018; 41(9): 1938-1946, doi: 10.2337/dc18-0623, indexed in Pubmed: 30026335.

34. Rosenstock J, Marquard J, Laffel L, et al. Empagliflozin as Adjunctive to Insulin Therapy in Type 1 Diabetes: The EASE Trials. Diabetes Care. 2018; 41(12): 2560-2569, doi: 10.2337/dc18-1749, indexed in Pubmed: 30287422

35. Sands A, Zambrowicz B, Rosenstock J, et al. Sotagliflozin, a Dua SGLT1 and SGLT2 Inhibitor, as Adjunct Therapy to Insulin in Type 1 Diabetes. Diabetes Care. 2015; 38(7): 1181-1188, doi: 10.2337/dc14-2806, indexed in Pubmed: 26049551.

36. Shimada A, Hanafusa T, Yasui A, et al. Empagliflozin as adjunct to insulin in Japanese participants with type 1 diabetes: Results of a 4-week, double-blind, randomized, placebo-controlled phase 2 trial. Diabetes Obes Metab. 2018; 20(9): 2190-2199, doi: 10.1111/dom.13351, indexed in Pubmed: 29766633

37. Garber AJ. Treat-to-target trials: uses, interpretation and review of concepts. Diabetes Obes Metab. 2013; 16(3): 193-205, doi: 10.1111/dom.12129, indexed in Pubmed: 23668598.

38. Danne T, Nimri R, Battelino T, et al. International Consensus on Use of Continuous Glucose Monitoring. Diabetes Care. 2017; 40(12): 1631-1640, doi: 10.2337/dc17-1600, indexed in Pubmed: 29162583.

39. McKnight JA, Wild SH, Lamb M, et al. Glycaemic control of Type 1 diabetes in clinical practice early in the 21st century: an international comparison. Diabet Med. 2015; 32(8): 1036-1050, doi: 10.1111/dme.12676, indexed in Pubmed: 25510978.

40. Miller K, Foster N, Beck R, et al. Current State of Type 1 Diabetes Treatment in the U.S.: Updated Data From the T1D Exchange Clinic Registry. Diabetes Care. 2015; 38(6): 971-978, doi: 10.2337/dc15-0078, indexed in Pubmed: 25998289

41. Weinstock R, Schütz-Fuhrmann I, Connor C, et al. Type 1 diabetes in older adults: Comparing treatments and chronic complications in the United States T1D Exchange and the German/Austrian DPV registries. Diabetes Res Clin Pract. 2016; 122: 28-37, doi: 10.1016/j.diabres.2016.09.024, indexed in Pubmed: 27764721.

42. Wing RR, Lang W, Wadden TA, et al. Benefits of Modest Weight Loss in Improving Cardiovascular Risk Factors in Overweight and Obese Individuals With Type 2 Diabetes. Diabetes Care. 2011; 34(7): 1481-1486, doi: 10.2337/dc10-2415, indexed in Pubmed: 21593294.

43. Van Ga, Wauters MA, De Le. The beneficial effects of modest weight loss on cardiovascular risk factors. Int J Obes Relat Metab Disord . 1997; 21(Suppl 1): S5-S9, indexed in Pubmed: 9130034 